\title{
Absolutely continuous energy bands in the electronic spectrum of quasiperiodic ladder networks
}

\author{
Biplab Pa因 and Arunava Chakrabart团 \\ Department of Physics, University of Kalyani, Kalyani, West Bengal-741 235, India
}

\begin{abstract}
The energy spectra of quasi-one dimensional quasiperiodic ladder networks are analyzed within a tight binding description. In particular, we show that if a selected set of sites in each strand of a ladder is tunnel-coupled to quantum dots attached from a side, absolutely continuous subbands can be generated in the spectrum if one tunes the dot potential and the dot-strand coupling appropriately. Typical cases with two and three strand Fibonacci ladders in the off diagonal model are discussed in details. We also discuss the possibility of re-entrant insulator-metal transition for a general $n$-strand ladder network when $n$ becomes large. The observations remain valid even in the case of a disordered ladder network with the same constituents. The results are analytically exact.
\end{abstract}

PACS numbers: 71.30.+h,72.15.Rn,03.75.-b

Keywords: Aperiodic nanostructure, Metal-insulator transition, Charge transport

\section{INTRODUCTION}

Anderson localization [1] 3] of electronic states in a disordered system is a path breaking observation that has extended its realm well beyond the electronic properties of disordered solid materials, and has been found out to be ubiquitous in a wide variety of systems such as the photonic [4, 5], phononic [6, 7], polaronic [8, 9], or plasmonic lattices [10, 11 to name a few. Artificial, tailor made geometries developed using the improved fabrication and lithographic techniques have been instrumental in testing the validity of the basic theory of exciton localization in presence of disorder. Examples include the direct observation of localization of matter waves in recent times [12 14].

Localization is a result of quantum interference that has the strongest manifestation in one dimension where all the single particle states exhibit an exponential decay of amplitude as one moves away from a particular lattice point. For dimensions beyond one, the states retain their characteristic decay in amplitude, with the addition of a possibility of a metal-insulator transition in three dimensions. The results have been substantiated by calculations of the localization length 15, 16], density of states [17] and their multi-fractality [18, 19].

Extensive work has also revealed the intricacies of the single parameter scaling hypothesis 3], its validity [20] or, variance [21], and even violation [22 24] in low dimensional systems within a tight binding approximation. The studies have subsequently been consolidated by direct experimental measurements of conductance distribution in quasi-one dimensional gold wires [25].

In the last couple of decades variations in the canonical cases of Anderson localization have surfaced, particularly, within a tight binding description. Resonant tun-

*Electronic address: biplabpal@klyuniv.ac.in

$\dagger$ Electronic address: arunava_chakrabarti@yahoo.co.in Tel.: +91 33 25820184, Fax: +913325828282 nelling of electronic states has been shown to arise even in a one dimensional disordered system as a consequence of certain special kinds of positional correlation [26 29]. Discrete energy levels corresponding to extended eigenfunctions [26], appear within a typical point spectrum exhibited by the randomly disordered lattices rendering the two-terminal transport completely ballistic at special values of the Fermi level. Such results are common even to infinite quasicrystalline lattices in one dimension where, the self similar character of the chain really allows an infinite number of unscattered (extended) eigenfunctions despite the absence of any translational invariance [30].

In this context, rather unusual, yet meaningful question could be, is it possible to engineer, in a controlled fashion, an absolutely continuous portion in the energy spectrum of non-translationally invariant systems with the help of some kind positional correlations ? The existence of real continuous bands are reported recently in quasi one dimensional or two dimensional systems only with diagonal disorder [28, 29]. We thus have a partial answer to the question, and it remains to be seen whether such bands of extended eigenfunctions can be generated and controlled with off-diagonal disorder are well.

We address this issue with respect to quasiperiodically ordered geometries. In this communication we show that, in quasi-one dimensional ladder networks with offdiagonal quasi-periodic order it is possible to engineer absolutely continuous bands of energy eigenvalues. We specifically work with the Fibonacci quasiperiodic order, though the observation is by no means, restricted to them. Ladder networks within the framework of tight binding model have already been exploited to gain insight into charge transport in DNA [31], to unravel interesting physics related to polymer structures and ployacene lattice topology [32, 33]. Comparatively speaking, controlled generation of absolutely continuous portions in the energy spectrum of the ladder networks, disordered or quasi-periodic, still remains to be explored. If it works then such an effort may open up new physics, or allow one to explore the possibility of devising novel low dimensional filter networks. 
The central result of this paper is that, one can create absolutely continuous subbands in the electronic spectrum of such quasi 1-d systems, by attaching a single quantum dot (QD) from one side to a selected set of lattice points in every strand of the ladder. We discuss the results explicitly in terms of Fibonacci quasiperiodic two and three arm ladder network models using a tight binding Hamiltonian and a real space renormalization group (RSRG) method. We also investigate whether a crossover in the fundamental character of the eigenfunctions, going from a typical critical nature to a Bloch-like extended character can be engineered. As will be shown, this indeed turns out to be quite a possibility in the case of the quasi-periodic ladder network (QPLN), particularly when the number of strands ' $n$ ' in the transverse direction increases. The results hold even for an off-diagonally disordered ladder where a re-entrant insulator-metal-insulator transition becomes feasible.

The system is described by a tight binding Hamiltonian, and is depicted in Fig. 11 The on-site potential at every lattice point is kept constant, while the nearest neighbor hopping integral along the arms of the ladder assumes two values dictated by the growth rule of the desired quasi-periodic sequence. The electron can tunnel between the arms of the ladder by hopping from one site to its corresponding site on the other arm through the hopping integral $\xi$ (see Fig. 1). The sites marked by $\alpha$ in each arm of the ladder is coupled to a single level QD 34 attached from one side. The QD couples to every $\alpha$-site via a hopping integral $\lambda$. There is no positional correlation, short range or long range in the conventional sense [26, 27]. Attachment of QD's from a side can quench the conductance of a linear chain [35], but an array of QD's in a ladder can have reverse effect, as will be seen.

We will show in a completely analytical way that, for a particular relationship between the nearest neighbor hopping integrals along the ladder strand and the QD-strand coupling $\lambda$, and for a special value of the QD potential, we get absolutely continuous subbands in the spectrum. This immediately raises a non-trivial issue that, the nature of the electronic spectrum and the eigenstates over a continuous range of eigenvalues can become sensitive to the numerical values of the Hamiltonian parameters - a fact that is not commonly observed in the conventional Anderson localization problem. The full spectrum turns out to be an admixture of conventional critical eigenstates, characteristics of a Fibonacci quasiperiodic chain, and absolutely continuous parts, giving rise to the possibility of a phase transition, particularly when the system grows in the transverse direction. Our results are analytically exact.

In section II we describe the Hamiltonian and the basic working principle for the two and three strand quasiperiodic ladders constructed following a Fibonacci sequence. Along with this, the real space renormalization scheme to obtain the energy spectrum and to judge the character of the single particle states is also presented.
In section III we present a result for the two terminal transmission coefficient of a finite sized QPLN, and in section IV we draw our conclusion.

\section{THE MODEL AND THE METHOD}

In Wannier basis the tight binding Hamiltonian of a general $n$-arm ladder network reads 29],

$$
\boldsymbol{H}=\sum_{i} \epsilon_{i} c_{i}^{\dagger} c_{i}+\sum_{\langle i j\rangle} t_{i j}\left[c_{i}^{\dagger} c_{j}+h . c .\right]
$$

where, $\boldsymbol{\epsilon}_{\boldsymbol{i}}$ is, in general, an $n \times n$ on-site potential matrix at the $i$-th vertical rung. We shall explicitly discuss the two-strand ladder at first. The cases $n \geq 2$ can be trivially constructed. $\boldsymbol{c}_{\boldsymbol{i}}^{\dagger}\left(\boldsymbol{c}_{\boldsymbol{i}}\right)$ is the creation (annihilation) operator, represented by rows (columns) of appropriate dimension, and $\boldsymbol{t}_{\boldsymbol{i j}}$ is the $n \times n$ hopping matrix representing hopping along and in between strands of the ladder, $n$ being equal to 2, 3 or any integer.

The Fibonacci ladder network is grown by attaching identical one dimensional Fibonacci chains in the transverse direction. Each individual chain grows along the horizontal direction following the binary Fibonacci sequence of two letters $L$ and $S$ (representing two bonds). The growth rule is, $L \rightarrow L S$ and $S \rightarrow L$, and the sequence begins with $L$. The on-site potentials at the vertices in each strand are designated by three symbols viz., $\epsilon_{\alpha}, \epsilon_{\beta}$ and $\epsilon_{\gamma}$ for sites flanked by two $L$-bonds, $L$ on left and $S$ on right, and $L$ on right and $S$ on left respectively. The attached QD is shown as " $\mu$ " in Fig. 1, and the potential at the side coupled QD is designated by $\epsilon_{\mu}$. The nearest neighbor hopping integral $t_{i j}=t_{L}$ or $t_{S}$ along ladder-arms following the Fibonacci sequence. $t_{i j}=\xi$ is the inter-arm hopping, and $t_{i j}=\lambda$ designates the tunnel hopping between the QD and the $\alpha$-sites of the ladder network. In our work, we shall assume $\epsilon_{\alpha}=\epsilon_{\beta}=\epsilon_{\gamma}=\epsilon_{0}$, and $t_{L} \neq t_{S}$. So, this is purely a transfer model [36] in the standard language of one dimensional quasicrystals.

We shall analyze the problem using a method described earlier [28, 29] in which an ' $n$ '-strand ladder $(n=2$ in the first phase of our discussion) is decoupled, making a change of basis, into a system of $n$ independent linear chains. The complete spectrum of the quasi 1-d system of $n$ strands is then obtained by convolving the spectra of the individual linear chains. In addition, we perform an RSRG analysis, which does not need any decoupling and yields a density of states spectrum that is in conformity with our results obtained using the decoupling scheme. The two terminal transmission spectrum is also computed using the RSRG decimation scheme to highlight the effect of the side coupled QD's.

The analysis will be carried over to the three strand ladder, and a generalization to the $n$ strand case will be discussed using arguments. 


\section{A. The two-strand Fibonacci ladder}

1. The decoupling of the strands and the spectral analysis

At first, we renormalize the potential at the $\alpha$-sites by decimating the side coupled QD. This leads to a renormalized value of the on-site potential at the $\alpha$-vertex, given by, $\bar{\epsilon}_{\alpha}=\epsilon_{0}+\lambda^{2} /\left(E-\epsilon_{\mu}\right)$.

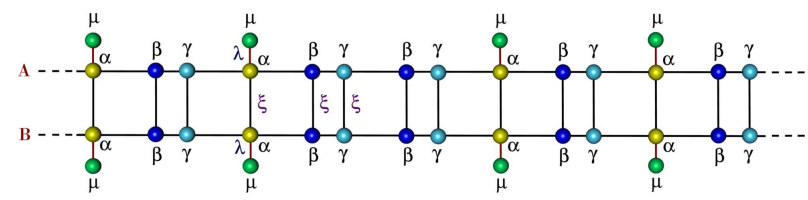

FIG. 1: (Color online) Typical realization of a two strand Fibonacci ladder. The sites $\alpha, \beta$ and $\gamma$ are colored differently to distinguish between the nearest neighbor bond-environments. The QD's $\mu$ are coupled to the $\alpha$-sites in each case. The coupling between $\mu$ and $\alpha$-site is denoted by $\lambda$ and the interstrand coupling is denoted by $\xi$.
The Schrödinger equation for the two-arm ladder can now be written equivalently in the form of the difference equations involving $2 \times 2$ matrices:

$$
\left(E I-\epsilon_{i}\right) \psi_{i}=\sum_{j} t_{i j} \psi_{j}
$$

where,

$$
\psi_{i}=\left(\begin{array}{c}
\psi_{i, A} \\
\psi_{i, B}
\end{array}\right)
$$

and,

$$
\boldsymbol{\epsilon}_{\boldsymbol{i}}=\left(\begin{array}{cc}
\epsilon_{i} & \xi \\
\xi & \epsilon_{i}
\end{array}\right)
$$

and, $\boldsymbol{I}$ is the $2 \times 2$ unit matrix. Written explicitly, the matrix equation reads,

$$
\left[\left(\begin{array}{cc}
E & 0 \\
0 & E
\end{array}\right)-\left(\begin{array}{cc}
\epsilon_{i} & \xi \\
\xi & \epsilon_{i}
\end{array}\right)\right]\left(\begin{array}{l}
\psi_{i, A} \\
\psi_{i, B}
\end{array}\right)=\left(\begin{array}{cc}
t_{i, i+1}^{A} & 0 \\
0 & t_{i, i+1}^{B}
\end{array}\right)\left(\begin{array}{c}
\psi_{i+1, A} \\
\psi_{i+1, B}
\end{array}\right)+\left(\begin{array}{cc}
t_{i, i-1}^{A} & 0 \\
0 & t_{i, i-1}^{B}
\end{array}\right)\left(\begin{array}{c}
\psi_{i-1, A} \\
\psi_{i-1, B}
\end{array}\right)
$$

In Eq. (5), the potential $\epsilon_{i}$ takes on values $\bar{\epsilon}_{\alpha}$ corresponding to a renormalized $\alpha$-site in each arm, while it is equal to $\epsilon_{0}$ for both the $\beta$ and $\gamma$-sites in each arm. The nearest neighbor hopping integrals $t_{i, i \pm 1}^{A(B)}$ are $t_{L}$ or $t_{S}$ depending on the local environment of the corresponding site in the $\operatorname{arm} A(B)$. A look at the Fig. 11will make it obvious that there are three such equations corresponding to the three different kinds of vertices viz., $\alpha, \beta$ and $\gamma$ respectively. The potential matrix $\boldsymbol{\epsilon}_{\boldsymbol{i}}$

corresponding to the renormalized $\alpha$-sites is,

$$
\left(\begin{array}{cc}
\epsilon_{0}+\frac{\lambda^{2}}{E-\epsilon_{\mu}} & \xi \\
\xi & \epsilon_{0}+\frac{\lambda^{2}}{E-\epsilon_{\mu}}
\end{array}\right) \equiv \boldsymbol{\epsilon}_{\mathbf{0}}+\frac{\lambda^{2}}{E-\epsilon_{\mu}} \boldsymbol{I}
$$

and that for the $\beta$ and $\gamma$ sites are

$$
\epsilon_{0}=\left(\begin{array}{cc}
\epsilon_{0} & \xi \\
\xi & \epsilon_{0}
\end{array}\right)
$$

We now diagonalize the $\boldsymbol{\epsilon}_{\mathbf{0}}$ matrix using a matrix $\boldsymbol{S}$. The matrix $\boldsymbol{S}$ makes a change over to a new basis defined by, $\phi_{\boldsymbol{i}}=\boldsymbol{S}^{-1} \boldsymbol{\psi}_{\boldsymbol{i}}$. As shown elsewhere [28, 29], such a change of basis leads to a decoupling of the set of Eq. (5) into two independent equations, one corresponding to the $A$-arm alone, and the other, to the arm $B$. Written explicitly, the equations read,

$$
\begin{aligned}
{[E-} & \left.\left(\epsilon_{0}+\xi+\frac{\lambda^{2}}{E-\epsilon_{\mu}}\right)\right] \phi_{i, A}=t_{L} \phi_{i+1, A}+t_{L} \phi_{i-1, A} \\
& {\left[E-\left(\epsilon_{0}+\xi\right)\right] \phi_{i, A}=t_{S} \phi_{i+1, A}+t_{L} \phi_{i-1, A} } \\
& {\left[E-\left(\epsilon_{0}+\xi\right)\right] \phi_{i, A}=t_{L} \phi_{i+1, A}+t_{S} \phi_{i-1, A} }
\end{aligned}
$$

for the sites $\alpha, \beta$ and $\gamma$ sequentially in $\operatorname{arm} A$. Similar set of equations for $\alpha, \beta$ and $\gamma$ sites in $\operatorname{arm} B$ will be,

$$
\begin{aligned}
{[E-} & \left.\left(\epsilon_{0}-\xi+\frac{\lambda^{2}}{E-\epsilon_{\mu}}\right)\right] \phi_{i, B}=t_{L} \phi_{i+1, B}+t_{L} \phi_{i-1, B} \\
& {\left[E-\left(\epsilon_{0}-\xi\right)\right] \phi_{i, B}=t_{S} \phi_{i+1, B}+t_{L} \phi_{i-1, B} } \\
& {\left[E-\left(\epsilon_{0}-\xi\right)\right] \phi_{i, B}=t_{L} \phi_{i+1, B}+t_{S} \phi_{i-1, B} }
\end{aligned}
$$

Needless to say, each of the sets Eq. (8) and Eq. (9) separately represents an independent Fibonacci chain. The spectrum of each chain bears the usual singular continuous character. The spectrum of the original ladder network can be obtained by convolution of the density of states of the individual decoupled Fibonacci chains. However, an analysis of these decoupled sub-systems yields rich spectral insight that would otherwise be difficult to obtain. We are now in a position to throw some light on this issue.

First, we need to appreciate that, in the new basis each decoupled Fibonacci chain is described by a set of three transfer matrices, viz., $\boldsymbol{M}_{\boldsymbol{\alpha}}, \boldsymbol{M}_{\boldsymbol{\beta}}$ and $\boldsymbol{M}_{\boldsymbol{\gamma}}$ [36]. For the $A$ arm decoupled Fibonacci chain, the explicit expressions 
of these matrices are

$$
\begin{aligned}
& M_{\boldsymbol{\alpha}, \boldsymbol{A}}=\left(\begin{array}{cc}
{\left[E-\left(\epsilon_{0}+\xi+\frac{\lambda^{2}}{E-\epsilon_{\mu}}\right)\right] / t_{L}} & -1 \\
1 & 0
\end{array}\right) \\
& \boldsymbol{M}_{\boldsymbol{\beta}, \boldsymbol{A}}=\left(\begin{array}{cc}
{\left[E-\left(\epsilon_{0}+\xi\right)\right] / t_{S}-t_{L} / t_{S}} \\
1 & 0
\end{array}\right) \\
& \boldsymbol{M}_{\boldsymbol{\gamma}, \boldsymbol{A}}=\left(\begin{array}{cc}
{\left[E-\left(\epsilon_{0}+\xi\right)\right] / t_{L}} & -t_{S} / t_{L} \\
1 & 0
\end{array}\right)
\end{aligned}
$$

respectively.

A similar set of transfer matrices for the decoupled $B$ arm reads,

$$
\begin{aligned}
& M_{\boldsymbol{\alpha}, \boldsymbol{B}}=\left(\begin{array}{cc}
{\left[E-\left(\epsilon_{0}-\xi+\frac{\lambda^{2}}{E-\epsilon_{\mu}}\right)\right] / t_{L}} & -1 \\
1 & 0
\end{array}\right) \\
& \boldsymbol{M}_{\boldsymbol{\beta}, \boldsymbol{B}}=\left(\begin{array}{cc}
{\left[E-\left(\epsilon_{0}-\xi\right)\right] / t_{S}-t_{L} / t_{S}} \\
1 & 0
\end{array}\right) \\
& \boldsymbol{M}_{\boldsymbol{\gamma}, \boldsymbol{B}}=\left(\begin{array}{cc}
{\left[E-\left(\epsilon_{0}-\xi\right)\right] / t_{L}} & -t_{S} / t_{L} \\
1 & 0
\end{array}\right)
\end{aligned}
$$

$\boldsymbol{M}_{\boldsymbol{\alpha}}$ and $\boldsymbol{M}_{\boldsymbol{\gamma} \boldsymbol{\beta}}=\boldsymbol{M}_{\boldsymbol{\gamma}} \cdot \boldsymbol{M}_{\boldsymbol{\beta}}$ follow an arrangement in the Fibonacci sequence 36.

Speaking in terms of the individual decoupled Fibonacci chains $A$ and $B$, we already know that each such chain offers a singular continuous spectrum. If we set $\lambda=0$, then each chain describes a separate Fibonacci lattice with $\epsilon_{\alpha}=\epsilon_{\beta}=\epsilon_{\gamma}=\epsilon_{0} \pm \xi$, and $t_{L} \neq t_{S}$. The total transfer matrix for any $m+1$-th generation of such a chain is related to its predecessors at the $m$ th and $m-1$ th generations by the matrix map $M_{m+1}=M_{m-1} \cdot M_{m}$, which will exhibit a six cycle [36] at $E=\epsilon_{0} \pm \xi$ for the decoupled $A$ and $B$ chains respectively. The existence of a six cycle of the matrix map implies that the above energy values are indeed eigenvalues of the infinite decoupled chains, and hence, the infinite ladder network as well.

Thus, the first fruit of the decoupling scheme is that, with $\lambda=0$ we obtain at least two eigenvalues which definitely belong to the spectrum of the infinite ladder. Extending this idea to an $n$-strand Fibonacci ladder we can easily identify $n$ different energy eigenvalues of the full quasi 1-d Fibonacci network in the absence of $\lambda$ (i.e. detaching the QD's) in a completely analytical way. The task becomes non-trivial when one consider the QPLN as a whole, without decoupling. Now let us look at the more serious effect when we include the QD by making $\lambda$ non-zero.

\section{The effect of $Q D$-strand coupling $(\lambda \neq 0)$}

Let us choose Eq. (10). We set $\epsilon_{\mu}=\epsilon_{0}+\xi$. It can easily be verified that, with this potential, the commutator $\left[\boldsymbol{M}_{\boldsymbol{\alpha}}, \boldsymbol{M}_{\boldsymbol{\gamma} \boldsymbol{\beta}}\right]=0$ for the decoupled $A$-arm, irrespective of the energy $E$ of the electron, if $\lambda=\sqrt{t_{S}^{2}-t_{L}^{2}}$. This implies that, with these conditions the individual $\alpha$-sites and the $\beta \gamma$ dimers in the decoupled $A$-arm can be arranged in any desired pattern, for example, in a perfectly periodic pattern. The spectrum offered by the decoupled $A$-arm Fibonacci chain will thus, under this choice of the QD potential $\epsilon_{\mu}$ and the tunnel hopping $\lambda$, will turn out to be indistinguishable from that of a perfectly periodic sequence of the $\alpha$-site (the 'renormalized' $\alpha$-site, to be precise) and the $\beta \gamma$ doublet. However, this argument needs to go through an acid test which we discuss below. Owing to the commutation of $\boldsymbol{M}_{\boldsymbol{\alpha}}$ and $\boldsymbol{M}_{\boldsymbol{\gamma}} \cdot \boldsymbol{M}_{\boldsymbol{\beta}}$, the de-

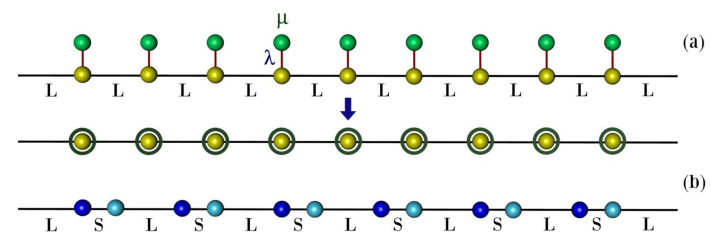

FIG. 2: (Color online) (a) The periodic $\alpha+$ QD chain, and its renormalized version. (b) The $\beta \gamma$ binary ordered chain. The LDGF is found at the site on the horizontal line in each case. The QD is renormalized to produce an effective one dimensional periodic chain of $\bar{\alpha}$ sites, as already discussed in the text.

coupled $A$-arm can be thought to be composed of two infinite periodic chains, one with $\alpha$-sites coupled to the QD's, and the other, a periodic array of $\beta \gamma$ clusters. The two chains are shown in Fig. 2, Each such infinite sublattice will have its own energy bands. It is important to verify whether, under the special condition imposed on the strand-dot coupling $\lambda$, the periodic $\alpha$-lattice and the periodic $\beta \gamma$ lattice share the same eigenvalue spectrum. If yes, then we conclusively prove the existence of an absolutely continuous part in the energy spectrum of the original ladder network.

To check this, we first of all construct an effective one dimensional chain composed of renormalized $\alpha$-atoms, obtained by decimating the side coupled dot. The resulting chain is illustrated in Fig. 2(a) with encircled lattice points. Each such lattice point has the renormalized onsite potential equal to $\bar{\epsilon}_{\alpha}=\epsilon_{0}+\xi+\lambda^{2} /\left(E-\epsilon_{\mu}\right)$. Each atom in the $\beta \gamma$ chain has the potential $\epsilon_{0}+\xi$. We now evaluate the local density of states (LDOS) at any site of the renormalized (encircled) $\alpha$-chain ( let's call it the $\bar{\alpha}$ chain ) and any site in the periodic $\beta \gamma$ chain (Fig. 2(b)). The local densities of states are given, for these two infinite periodic lattices, and for a given set values of $\epsilon_{0}$, $\xi, t_{L}$ and $t_{S}$, by $\rho_{\bar{\alpha}}(E)=(-1 / \pi)\left[\mathcal{F}_{\alpha}\left(E, \lambda, \epsilon_{\mu}\right)\right]^{-1 / 2}$ and 
$\rho_{\beta \gamma}=(-1 / \pi)\left[\mathcal{G}_{\beta \gamma}(E)\right]^{-1 / 2}$, where,

$$
\mathcal{F}_{\alpha}\left(E, \lambda, \epsilon_{\mu}\right)=4 t_{L}^{2}-\left(E-\epsilon_{0}-\xi-\frac{\lambda^{2}}{E-\epsilon_{\mu}}\right)^{2}
$$

and,

$$
\mathcal{G}_{\beta \gamma}(E)=\frac{4 t_{L}^{2} t_{S}^{2}}{\left(E-\epsilon_{0}-\xi\right)^{2}}-\left(E-\epsilon_{0}-\xi-\frac{t_{L}^{2}+t_{S}^{2}}{E-\epsilon_{0}-\xi}\right)^{2}
$$

If we now set $\epsilon_{\mu}=\epsilon_{0}+\xi$, then a simple algebra yields that, the difference $\Delta=\mathcal{G}_{\beta \gamma}-\mathcal{F}_{\alpha}$ is given by,

$$
\Delta=\frac{\left[2\left(E-\epsilon_{0}-\xi\right)^{2}+t_{L}^{2}-t_{S}^{2}-\lambda^{2}\right]\left(\lambda^{2}+t_{L}^{2}-t_{S}^{2}\right)}{\left(E-\epsilon_{0}-\xi\right)^{2}}
$$

which clearly vanishes for any value of the energy of the electron the moment we tune $\lambda=\sqrt{t_{S}^{2}-t_{L}^{2}}$. Thus the LDOS's of the two basic constituent periodic chains become identical independent of energy, resulting in a complete overlap of the spectra of these individual chains under the above resonance condition. As each of the $\alpha$ and the $\beta \gamma$ sublattices provide a two subband continuum in their energy spectra (an obvious effect of the binary periodic lattice), it is obvious that these two spectral continua will retain their positions in the full spectrum of the Fibonacci QPLN when the QD potential and the QDstrand tunnel coupling are tuned to the above special values.

It should be appreciated that once we fix $\epsilon_{\mu}=\epsilon_{0}+\xi$, the set of Eq. (11) corresponding to the decoupled $B$ strand, represents a mixed model linear Fibonacci chain. Its contribution to the full energy spectrum is the usual fragmented, multi-fractal one. As the full spectrum of the two-arm ladder will be a convolution of both, we expect it to have absolutely continuous parts decorated with a spiky envelope, and a mixture of extended Blochlike states and the critical eigenfunctions which are characteristic of a quasi-periodic Fibonacci sequence of potentials. This is precisely what we observe in Fig. 3(a).

At this point one must be careful to interpret Fig. 3(a). In the full energy spectrum, critical and extended eigenstates can never coexist. So, even in the full spectrum of the un-decoupled QPLN the entire range shown in Fig. 3(a) by grey shade corresponds to extended Blochlike states only. Beyond it, every wave function will be critical in nature. The contributions coming from each decoupled strand are only shown separately in this figure.

\section{The RSRG analysis}

The above arguments are confirmed by an exact real space renormalization group (RSRG) calculation of the local density of states, first of each individual decoupled strands and then of the full two-arm Fibonacci ladder without decoupling it. The first task is already discussed in literature [37, 38], and need not be talked about again in here. We instead, provide the details of the RSRG scheme of the full ladder network. We renormalize the full two-strand ladder by decimating the $\beta$-vertices. This generates new hopping integrals across the diagonals of the fundamental rectangular plaquettes of renormalized ladder denoted by $d_{L}^{\prime}$ and $d_{S}^{\prime}$ in the equations to follow. To implement the decimation we need to assign three different symbols to the inter-arm hopping $\xi$, viz., $\xi_{\alpha}$, $\xi_{\beta}$ and $\xi_{\gamma}$ connecting the $\alpha, \beta$ and $\gamma$ vertices of the two strands. Of course, we begin with $\xi_{\alpha}=\xi_{\beta}=\xi_{\gamma}=\xi$, and $d_{L}=d_{S}=0$. The recursion relations arising out of this decimation procedure are given by,

$$
\begin{aligned}
\bar{\epsilon}_{\alpha}^{\prime} & =\epsilon_{\gamma}+\mathcal{A} t_{L}+\mathcal{B} t_{S}+\mathcal{C} d_{L}+\mathcal{D} d_{S} \\
\epsilon_{\beta}^{\prime} & =\epsilon_{\gamma}+\mathcal{B} t_{S}+\mathcal{D} d_{S} \\
\epsilon_{\gamma}^{\prime} & =\bar{\epsilon}_{\alpha}+\mathcal{A} t_{L}+\mathcal{C} d_{L} \\
t_{L}^{\prime} & =\mathcal{B} t_{L}+\mathcal{D} d_{L} \\
t_{S}^{\prime} & =t_{L} \\
d_{L}^{\prime} & =\mathcal{B} d_{L}+\mathcal{D} t_{L} \\
d_{S}^{\prime} & =d_{L} \\
\xi_{\alpha}^{\prime} & =\xi_{\gamma}+\mathcal{A} d_{L}+\mathcal{B} d_{S}+\mathcal{C} t_{L}+\mathcal{D} t_{S} \\
\xi_{\beta}^{\prime} & =\xi_{\gamma}+\mathcal{B} d_{S}+\mathcal{D} t_{S} \\
\xi_{\gamma}^{\prime} & =\xi_{\alpha}+\mathcal{A} d_{L}+\mathcal{C} t_{L}
\end{aligned}
$$

where

$$
\begin{aligned}
\mathcal{A} & =\frac{\left(E-\epsilon_{\beta}\right) t_{L}+\xi_{\beta} d_{L}}{\mathcal{W}} \\
\mathcal{B} & =\frac{\left(E-\epsilon_{\beta}\right) t_{S}+\xi_{\beta} d_{S}}{\mathcal{W}} \\
\mathcal{C} & =\frac{\left(E-\epsilon_{\beta}\right) d_{L}+\xi_{\beta} t_{L}}{\mathcal{W}} \\
\mathcal{D} & =\frac{\left(E-\epsilon_{\beta}\right) d_{S}+\xi_{\beta} t_{S}}{\mathcal{W}} \\
\mathcal{W} & =\left(E-\epsilon_{\beta}\right)^{2}-\xi_{\beta}^{2} \\
\bar{\epsilon}_{\alpha} & =\epsilon_{0}+\lambda^{2} /\left(E-\epsilon_{\mu}\right)
\end{aligned}
$$

A small imaginary part is added to the energy $E$, and the LDOS at an $\alpha, \beta$ or $\gamma$ site is obtained by calculating the respective local Green's function $G_{00}^{i}=\left(E-\epsilon_{i}^{*}\right)^{-1}$, where $i=\alpha, \beta$ or $\gamma \cdot \epsilon_{i}^{*}$ represents the fixed point of the respective on-site potential as the hopping integrals flow to zero under the RSRG iterations. The LDOS is given by,

$$
\rho_{i}=-\frac{1}{\pi} \operatorname{Im}\left[G_{00}^{i}\right]
$$

The results are presented in Fig. 3. In panel (a) the spectra of the decoupled $A$ - and $B$-arms are shown separately, while panel (b) illustrates the spectrum of the full two-arm ladder as obtained from the RSRG study. The existence of the absolutely continuous portions in the energy spectrum is obvious. The character of the eigenfunctions belonging to the spectrum is checked from an observation of the flow of the the hopping integrals $t_{L}, t_{S}$ and 
the diagonal hopping amplitude under RSRG iterations. For any energy eigenvalue, chosen from any portion of the absolutely continuous sub-bands (grey shaded portion in Fig. 3(a)) all the hopping integrals, as obtained from Eq. (15), remain non-zero for an arbitrarily large number of iterations confirming the extended character of the wave functions. Beyond the extended regime, the hopping integrals flow to zero under iteration indicating that the eigenfunctions are not extended. In Fig. (3) b) we see the full LDOS spectrum from the coupled two-strand QPLN obtained from the RSRG method (using the set of Eq. (15)). The light blue shade in the spectrum is just to highlight the region of non-zero LDOS. The extended eigenstates occupy the precise positions as depicted by the grey shaded part in Fig. 3(a). The eigenstates form a mixed spectrum of critical and extended states, as one traverses the energy axis from the left.
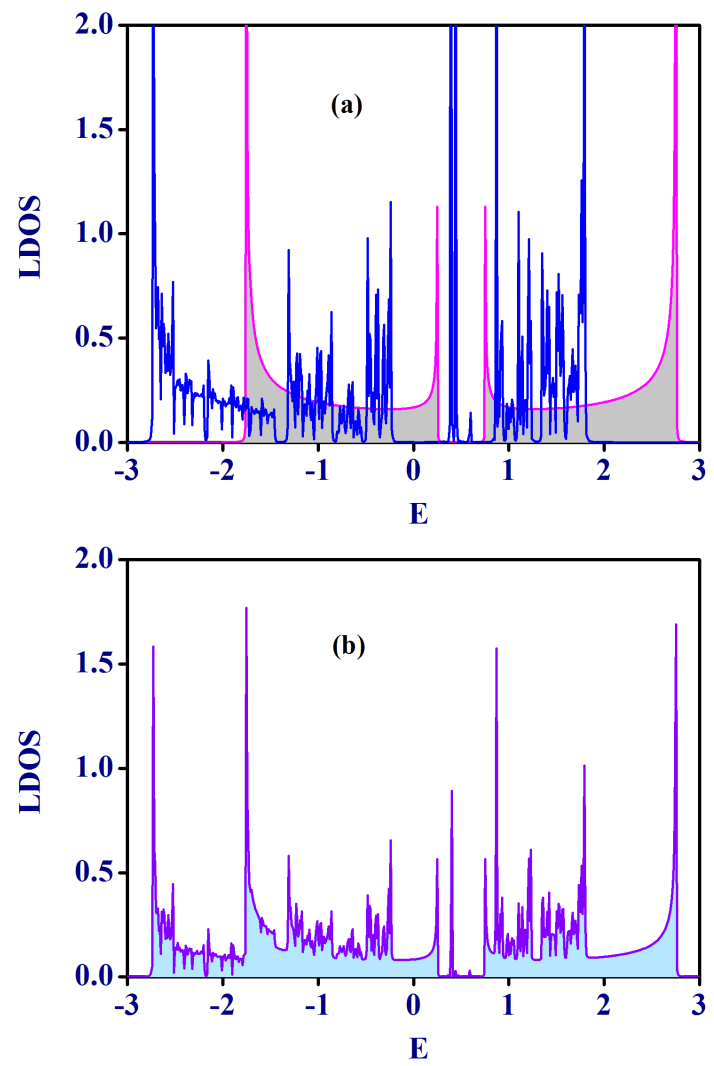

FIG. 3: (Color online) Plot of local density of states (LDOS) at an $\alpha$-vertex for a two-strand ladder network. The upper panel shows the LDOS spectra of the decoupled individual linear chains, one chain satisfying resonance condition. The lower panel shows the LDOS spectrum of the composite ladder network as obtained from the RSRG scheme, under resonance condition. The values of the parameters chosen are $\epsilon_{\alpha}=\epsilon_{\beta}=$ $\epsilon_{\gamma}=0, \epsilon_{\mu}=0.5, t_{L}=1, t_{S}=1.25, \lambda=0.75$, and $\xi=0.5$, measured in unit of $t_{L}$.

An additional support in regard of the continuous subbands and the extended eigenstates is also obtained from an observation of the flow pattern of the entire parameter space of the decoupled strands that satisfies the condition $\epsilon_{\mu}=\epsilon_{0}+\xi$ and $\lambda=\sqrt{t_{S}^{2}-t_{L}^{2}}$. For such a choice, under successive RSRG step, we always get $\bar{\epsilon}_{\alpha}^{(j)} \neq \epsilon_{\beta}^{(j)}=\epsilon_{\gamma}^{(j)}$ and $t_{L}^{(j)} \neq t_{S}^{(j)}$ for the decoupled $A$-strand. This is a typical flow in the parameter space that corresponds to the extended eigenstates in quasiperiodic Fibonacci chains [39]. In the present case it confirms the extended Bloch-like character of the wave function for the entire energy regime (given by the grey color in Fig. 3(a)).

\section{B. Three-arm Fibonacci ladder and beyond}

The motivation behind extending the above analysis to a three-arm Fibonacci ladder stems from the curiosity

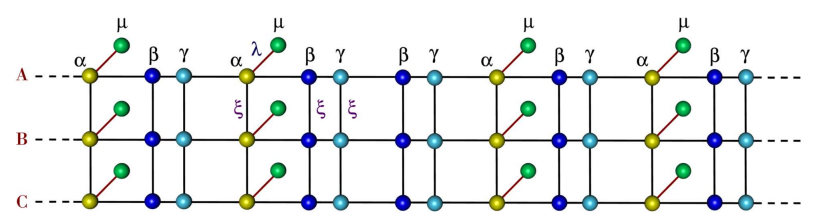

FIG. 4: (Color online) Schematic diagram of a three-strand Fibonacci ladder network. Meaning of all other symbols are same as in figure 1

to know whether, as one extends the ladder network in the transverse direction, any possibility of a re-entrant transition (or, crossover) from a singular continuous to an absolutely continuous energy spectrum becomes apparent. For disordered ladders comprising the same $\alpha$ and $\beta \gamma$ clusters this would imply the possibility of an insulator-metal transition that can be triggered by an appropriate choice of the numerical values of the QD potential $\epsilon_{\mu}$ and the tunnel hopping integral $\lambda$.

To check such a possibility we decouple a three-arm Fibonacci ladder (Fig. (4). We follow the same prescription for decoupling as outlined in the case of a two-strand ladder. Diagonalization of the potential matrix $\boldsymbol{\epsilon}_{\boldsymbol{i}}$ now yields three eigenvalues, viz., $\epsilon_{0}$ and $\epsilon_{0} \pm \sqrt{2} \xi$, and the effective potential at the $\alpha$-site in each decoupled arm will be $\epsilon_{0}+\lambda^{2} /\left(E-\epsilon_{\mu}\right)$ (for arm $A$, say), $\epsilon_{0}+\sqrt{2} \xi+\lambda^{2} /\left(E-\epsilon_{\mu}\right)$ (for arm $B$, say) and $\epsilon_{0}-\sqrt{2} \xi+\lambda^{2} /\left(E-\epsilon_{\mu}\right.$ ) (for arm $C)$. We now end up having three sets of equations, one for each decoupled strand. For example,

$$
\begin{gathered}
{\left[E-\left(\epsilon_{0}+\frac{\lambda^{2}}{E-\epsilon_{\mu}}\right)\right] \phi_{i, A}=t_{L} \phi_{i+1, A}+t_{L} \phi_{i-1, A}} \\
\left(E-\epsilon_{0}\right) \phi_{i, A}=t_{S} \phi_{i+1, A}+t_{L} \phi_{i-1, A} \\
\left(E-\epsilon_{0}\right) \phi_{i, A}=t_{L} \phi_{i+1, A}+t_{S} \phi_{i-1, A}
\end{gathered}
$$

corresponding to the $\alpha, \beta$ and $\gamma$ sites respectively in the decoupled $A$-strand. Two sets of similar equations are also obtained for the decoupled $B$ - and $C$-strands, with the effective potential at the $\alpha$-site being equal to $\epsilon_{0}+$ $\sqrt{2} \xi+\lambda^{2} /\left(E-\epsilon_{\mu}\right)$ and $\epsilon_{0}-\sqrt{2} \xi+\lambda^{2} /\left(E-\epsilon_{\mu}\right)$ respectively. The effective potentials at the $\beta$ and $\gamma$ sites in the $B$ and $C$ strand are $\epsilon_{0} \pm \sqrt{2} \xi$ as there is no side coupling to these sites. 
If, as before, we set $\epsilon_{\mu}=\epsilon_{0}$ in Eq. (17), then $\left[\boldsymbol{M}_{\boldsymbol{\alpha}}, \boldsymbol{M}_{\boldsymbol{\gamma} \boldsymbol{\beta}}\right]=0$ for the decoupled $A$-strand. The contribution to the full spectrum coming from this separate chain will be two absolutely continuous sub-bands. With the potential of the QD set at $\epsilon_{\mu}=\epsilon_{0}$, the two other decoupled strands represent two different Fibonacci chains in the mixed model. The spectrum resulting from each of them will remain fragmented Cantor type. The overall spectrum, as we now have known, will be a convolution of the three. The absolutely continuous portions will still hold their positions in the main spectrum, and will be flanked by the spiky, fragmented parts characteristics of critical eigenstates of a Fibonacci lattice (Fig [5). The eigenfunctions belonging to the continuous sub-bands will, needless to say, be of extended character.

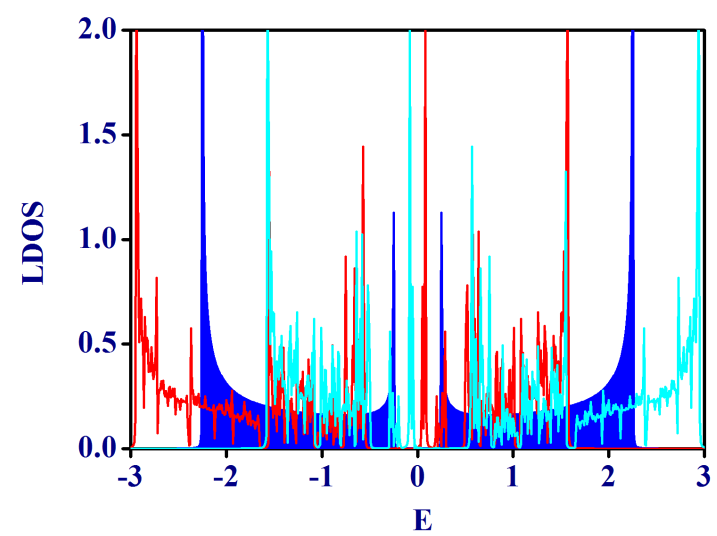

FIG. 5: (Color online) Plot of LDOS for a three-strand Fibonacci ladder network. It represents the LDOS spectra of the three decoupled isolated chains, one satisfying resonance condition (in blue shaded color) and the other two with offresonance condition (in red and cyan color). The parameters chosen here are same as in Figure 3

One needs to appreciate that each spectrum offered separately by the decoupled $B$ - and $C$-strands will have different "band-centres" around which the singular continuous spectra of the individual decoupled Fibonacci chain will spread out. With an $n$-strand ladder model, there will be $n-1$ such centers from the $n-1$ decoupled strands around which Cantor set energy eigenvalues will be distributed, the remaining strand contributing the absolutely continuous sub-bands. The centres will be densely populated around the outer edge of the continuous sub-bands as $n \rightarrow \infty$. Thus, with increasing number of strands there is a possibility of observing a smooth critical to extended state crossover in the spectrum of such quasi 1-d aperiodic ladder models. Clearly, the argument prevails even if we have an $n$-strand off diagonally disordered ladder network. The side coupled QD's with appropriately tuned potential one can then expect a re-entrant insulator-metal transition.

Before leaving this discussion, it is pertinent to mention that, the commutaion of transfer matrices leading to a complete, energy independent delocalization of electronic states have been reported recently in certain special examples of quasi-one dimensional lattices [40]. But, those systems do not open up any possibility of an insulator-metal transition, in the current spirit of the work. At the same time, it is useful to note that, the commutivity of transfer matrices has been used in the past to unravel discrete unscattered eigenstates in an array of quantum wells 41] or a disordered distribution of Pöschl-Teller potential [42]. The difference with the present communication and these two latter references lies in the energy-independent character of the matrixcommutation that plays the key role.

\section{TWO TERMINAL TRANSMISSION}

We now present partial results (to save space) for the two terminal transmission coefficient of a finite two strand ladder without decoupling it. No change of basis is made, and the full finite QPLN is treated within the RSRG scheme. The basic working formula is already elaborated in the existing literature 43]. The ladder is clamped between two semi-infinite perfectly ordered leads (viz., the source and the drain) at any two extremities (Fig. 6). The leads are characterized by uniform

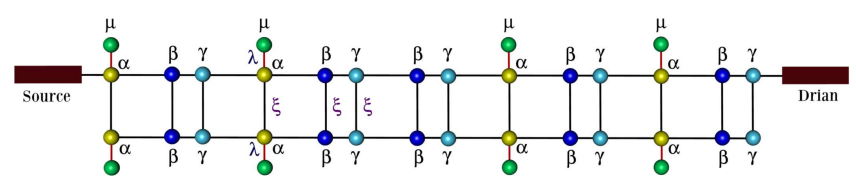

FIG. 6: (Color online) Schematic view of a finite size twostrand QPLN clamped between the source and the drain.

on-site potential $\epsilon_{\mathcal{L}}=0$ and nearest neighbor hopping integral $\tau_{\mathcal{L}}=2$. The portion of the ladder clamped between the leads is renormalized to bring it down to a $d i$ atomic molecule, and the transmission coefficient is easily obtained [4, 45]. The procedure is standard and to save space again, we do not provide the details.

It should be appreciated that, the precise details of the transmission coefficient definitely depends on which points in the ladder the leads are attached to. Only one result is shown here in which the two semi-infinite ordered leads are connected to the extreme points of the upper strand of a 10-th generation Fibonacci ladder having 89 bonds in each arm. One needs to appreciate that, even in the absence of any attached QD, a two strand finite QPLN can exhibit high transmission spikes with $T$ close to unity. This normally results from the finite size of the system, as well as from the fact that a ladder network consists of loops that brings in a flavor of long range connection, and may well be responsible for quantum interference leading to high transmittivity at selected values of electron-energy. Also, the attachment to the leads, and the difference between the hopping integral in the lead and that in the bulk of the system give rise to strong scattering effects at the contacts, affecting 


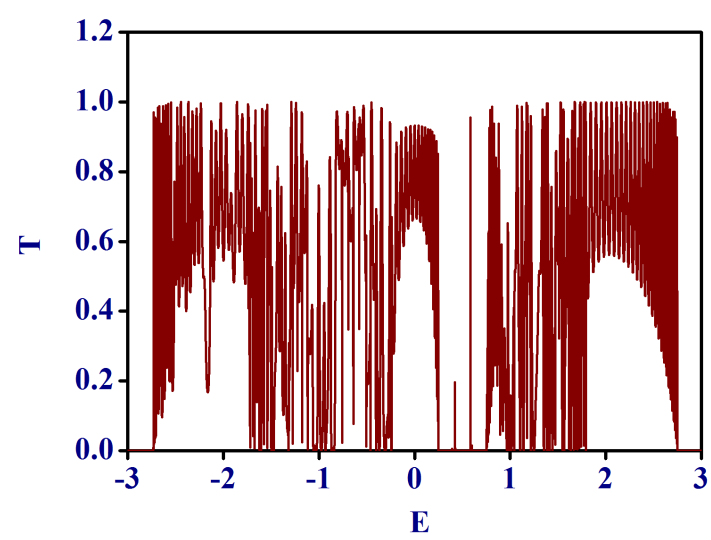

FIG. 7: (Color online) Transmission characteristics of a 10-th generation two-strand Fibonacci ladder network under resonance condition. Both the incoming and outgoing leads, viz., the source and the drain are connected in the upper strand. System parameters chosen are $\epsilon_{\alpha}=\epsilon_{\beta}=\epsilon_{\gamma}=0, \epsilon_{\mu}=0.5$, $t_{L}=1, t_{S}=1.25, \lambda=0.75$, and $\xi=0.5$, and the lead parameters are $\epsilon_{\mathcal{L}}=0$ and $\tau_{\mathcal{L}}=2$.

the overall transmission of the QPLN system. Therefore, the results obtained from a finite size analysis should be carefully compared with the predictions which come from the anatomy of an infinite system.

Nevertheless, the existence of the continuous parts in the energy spectrum of the infinite system is already showing up in the enhanced value of the transmission coefficient at appropriate values of the electron energy as can be seen in Fig. 7. The apparent drop to close to zero value at certain regions even within the continuous zone is due to the scale at which the transmission coefficient is shown here. However, the transmission coefficient exhibits wide fluctuations owing to the quasiperiodic nature of network. The effect of quantum interference is manifestly observed here. The finite value of the transmission coefficient even beyond the absolutely continuous part of the spectrum is due to the finite size of the system, and gets lower and lower, and the spectrum gets more spiky with increasing system size. Also, one can anticipate eigenstates with their 'localization length' much larger compared to the size of the system as considered in the present calculation.

\section{CONCLUDING REMARKS}

We have shown that Fibonacci quasi-periodic ladder networks, where quasi-periodic order is introduced in the distribution of bonds, keeping the potential at every vertex same, are capable of producing absolutely continuous portions in their energy spectra. This requires the attachment of quantum dots from one side to a special set of sites in each arm of the ladder. A suitable adjustment of the dot potential, together with the dotbackbone coupling can generate absolutely continuous energy sub-bands, decorated at the flanks by the usual critical states - a characteristic of quasi-periodic lattices. Apart from opening up the possibility of engineering the transport of excitons in such multi-strand systems, a very basic issue of Anderson localization is reviewed. It seems that, the existence of localized eigenstates in similar disordered ladder networks may strongly depend on the numerical values of the parameters in the Hamiltonian.

The analysis is carried over to other quasi-periodically ordered systems, such as the copper mean chain, the aperiodic Thue Morse chain and even to the disordered ladder networks with locally and non-locally coupled dots. The conditions for the existence of a continuum in the energy spectrum becomes much more non-trivial in such systems. These issues will be reported in a forthcoming article.

\section{Acknowledgments}

B.P. is grateful to DST, India for an INSPIRE Fellowship. A.C. acknowledges financial support from DST, India through a PURSE grant, and The Abdus Salam International Centre for Theoretical Physics, Trieste, Italy for its hospitality and financial support.
[1] P. W. Anderson, Phys. Rev. 109, 1492 (1958).

[2] B. Kramer and A. MacKinnon, Rep. Prog. Phys. 56, 1469 (1993).

[3] E. Abrahams, P. W. Anderson, D. C. Liciardello, and T. V. Ramakrishnan, Phys. Rev. Lett. 42, 673 (1979).

[4] E. Yablonovitch, Phys. Rev. Lett. 58, 2059 (1987).

[5] S. John, Phys. Rev. Lett. 58, 2486 (1987).

[6] F. R. Montero de Espinosa, E. Jiménez, and M. Torres, Phys. Rev. Lett. 80, 1208 (1998).

[7] J. O. Vasseur, P. A. Deymier, G. Frantziskonis, G. Hong, B. Djafari-Rouhani, and L. Dobrzynski, J. Phys.: Condens. Matter 10, 6051 (1998).

[8] I. O. Barinov, A. P. Alodzhants, and S. M. Arakelyan,
Quantum Electron. 39, 685 (2009).

[9] M. Grochol and C. Piermarocchi, Phys. Rev. B 78, 035323 (2008).

[10] A. Tao, P. Sinsermsuksaul, and P. Yang, Nature Nanotechnol. 2, 435 (2007).

[11] A. Christ, Y. Ekinci, H. H. Solak, N. A. Gippus, S. G. Tikhodeev, and O. J. F. Martin, Phys. Rev. B 76, 201405 (2007).

[12] B. Damski et al., Phys. Rev. Lett. 91, 080403 (2003).

[13] J. Billy et al., Nature (London) 453, 891 (2008).

[14] G. Roati et al., Nature (London) 453, 895 (2008).

[15] R. A. Römer and H. Schulz-Baldes, Europhys. Lett. 68, 247 (2004). 
[16] A. Eilmes, R. A. Römer, and M. Schreiber, Physica B 296, 46 (2001).

[17] A. Rodríguez, J. Phys. A: Math. Gen. 39, 14303 (2006).

[18] A. Rodriguez, L. J. Vasquez, and R. A. Römer, Phys. Rev. B 78, 195107 (2008).

[19] A. Rodriguez, L. J. Vasquez, K. Slevin, and R. A. Römer, Phys. Rev. B 84, 134209 (2011).

[20] S. D. Pinski, W. Schirmacher, and R. A. Römer, Europhys. Lett. 97, 16007 (2012).

[21] Lev I. Deych, A. A. Lisyanski, and B. L. Alshuler, Phys. Rev. Lett. 84, 2678 (2000).

[22] M. Titov and H. Schomerus, Phys. Rev. Lett. 95, 126603 (2005).

[23] J. W. Kantelhardt and A. Bunde, Phys. Rev. B 66, 035118 (2002).

[24] V. E. Kravtsov and V. I. Yudson, Phys. Rev. B 82, 195120 (2010)

[25] P. Mohanty and R. A. Webb, Phys. Rev. Lett. 88, 146601 (2002).

[26] D. H. Dunlap, H.-L. Wu, and P. Phillips, Phys. Rev. Lett. 65, 88 (1990).

[27] F. A. B. F. de Moura and M. Lyra, Phys. Rev. Lett. 81, 3735 (1998).

[28] S. Sil, S. K. Maiti, and A. Chakrabarti, Phys. Rev. B 78, 113103 (2008).

[29] A. Rodriguez, A. Chakrabarti, and R. A. Römer, Phys. Rev. B 86, 085119 (2012).

[30] A. Chakrabarti, S. N. Karmakar, and R. K. Moitra, Phys. Rev. B 50, 13276 (1994).
[31] E. Maciá, Phys. Rev. B 74, 245105 (2006); Phys. Rev. B 80, 125102 (2009)

[32] M. Bravi, R. Farchioni, G. Grosso, and G. P. Parravicini, Phys. Rev. B 87, 035105 (2013).

[33] R. Farchioni, G. Grosso, and G. P. Parravicini, Eur. Phys. J. B 84, 227 (2011).

[34] B. Kubala and J. König, Phys. Rev. B 67, 205303 (2003).

[35] R. Farchioni, G. Grosso, and G. P. Parravicini, Phys. Rev. B 85, 165115 (2012).

[36] M. Kohmoto, B. Sutherland, and C. Tang, Phys. Rev. B 35, 1020 (1987).

[37] J. A. Ashraff and R. B. Stinchcombe, Phys. Rev. B 37, 5723 (1988).

[38] A. Chakrabarti, S. N. Karmakar, and R. K. Moitra, Phys. Rev. B 39, 9730 (1989).

[39] A. Chakrabarti, S. N. Karmakar, and R. K. Moitra, Phys. Lett. A 168, 301 (1992).

[40] B. Pal, S. K. Maiti, and A. Chakrabarti, Europhys. Lett. 102, 17004 (2013).

[41] I. Gómez, F. Dóminguez-Adame, and E. Diez, Physica B 324, 235 (2002).

[42] A. Rodríguez and J. M. Cerveró, Phys. Rev. B 74, 104201 (2006).

[43] A. D. Stone, J. D. Joannopoulos, and D. J. Chadi, Phys. Rev. B 24, 5583 (1981).

[44] A. Chakrabarti, Phys. Rev. B 74, 205315 (2006).

[45] B. Pal and A. Chakrabarti, Eur. Phys. J. B 85, 307 (2012). 